\title{
V4332 Sagittarii revisited ${ }^{\star}$
}

\author{
R. Tylenda ${ }^{1,3}$, L. A. Crause ${ }^{2}$, S. K. Górny ${ }^{1}$, and M. R. Schmidt ${ }^{1}$ \\ 1 Department for Astrophysics, N. Copernicus Astronomical Centre, Rabiańska 8, 87-100 Toruń, Poland \\ e-mail: tylenda@ncac.torun.pl \\ 2 South African Astronomical Observatory, PO Box 9, Observatory 7935, South Africa \\ 3 Centre for Astronomy, N. Copernicus University, Torun, Poland
}

Received 2 July 2004 / Accepted 8 May 2005

\begin{abstract}
The eruption of V4332 Sgr discovered in February 1994 shows striking similarities to that of V838 Mon started in January 2002. The nature of these eruptions is, however, enigmatic and unclear. We present new photometric and spectroscopic data on V4332 Sgr obtained in April-May 2003 at the SAAO. The obtained spectrum shows an unusual emission-line component superimposed on an early M-type stellar spectrum. The emission-line spectrum is of very low excitation and is dominated by lines from neutral elemets (NaI, FeI, CaI) and molecular bands ( $\mathrm{TiO}, \mathrm{ScO}, \mathrm{AlO}$ ). We also analyse all the observational data, mainly photometric measurements, available for V4332 Sgr. This allows us to follow the evolution of the effective temperature, radius and luminosity of the object since February 1994 till 2003. We show that the observed decline of V4332 Sgr can be accounted for by a gravitational contraction of an inflated stellar envelope. The combined optical and infrared photometry in 2003 shows that apart from the M-type stellar component there is a strong infrared excess in the $K L M$ bands. This excess was absent in the 2MASS measurements done in 1998 but was probably starting to appear in $K$ in 1999 when the object was observed in the DENIS survey. We interpret the results in terms of a stellar merger scenario proposed by Soker \& Tylenda. The infrared excess is likely to be due to a disc-like structure which is either of protostellar nature or has been produced during the 1994 eruption and stores angular momentum from the merger event.
\end{abstract}

Key words. stars: variables: general - stars: circumstellar matter - stars: fundamental parameters stars: individual: V4332 Sgr, V838 Mon

\section{Introduction}

V4332 Sagittarii was discovered towards the end of February 1994 as an apparent nova. Subsequent spectroscopic observations however indicated that this was not a classical nova. It lacked spectral features characteristic of novae while its spectral type, K3-4 III-I on March 4, was too late for a nova at maximum and with time, contrary to classical novae, it evolved toward lower and lower effective temperatures. A detailed description and analysis of available observational data for V4332 Sgr in eruption can be found in Martini et al. (1999, hereafter MWT99).

Similar evolution has been observed for V838 Mon which was discovered in eruption in early January 2002. This object underwent more complex photometric and spectral evolution, but in March-April 2002 its behaviour was quite similar to that observed for V4332 Sgr (e.g. Munari et al. 2002; Crause et al. 2003). Therefore V838 Mon and V4332 Sgr (and presumably also M31 RV - see Mould et al. 1990) are considered a mysterious new class of eruptive variables. As discussed in

^ Based on observations made at the South African Astronomical Observatory.
Soker \& Tylenda (2003), thermonuclear events, i.e. nova-type runaway or late He-shell flash, cannot account for the observed evolution of these objects. Instead these authors propose that the eruptions can be due to stellar merger events (see also Retter \& Marom 2003).

V4332 Sgr has been forgotten by observers since its decline in June 1994. As far as we know, the only observations obtained between 1994-2003 are 2MASS measurements in May 1998 and DENIS photometry in September 1999. Motivated by the interest in V838 Mon, we took low resolution optical spectra of V4332 Sgr in April 2003, followed by photomertic measurements in May 2003. We were surprised to find that the object displayed an unusual emission-line spectrum, completely different to that of typical emission-line objects such as planetary nebulae, symbiotic stars or T Tauri stars. The strongest emission feature appeared to belong to the $\mathrm{Na} I \mathrm{D}$ lines, but later we realised that many emission lines are due to molecular bands of $\mathrm{TiO}, \mathrm{AlO}$ and ScO. Banerjee et al. (2003, hereafter BVA03) observed V4332 Sgr in near-IR in April-June 2003. Their spectra also show strong emission features which they attribute to AlO. More recently, in September 2003, Banerjee \& Ashok (2004, hereafter BA04) observed the object in the optical and obtained 
Table 1. Cousins photometry of V4332 Sgr obtained on 21 May 2003.

\begin{tabular}{ccll}
\hline \hline UT & Band & Magnitude & Error \\
\hline $03: 50$ & $B$ & 19.6 & \pm 0.2 \\
$03: 15$ & $V$ & 17.63 & \pm 0.03 \\
$03: 27$ & $R_{\mathrm{c}}$ & 16.34 & \pm 0.00 \\
$03: 39$ & $I_{\mathrm{c}}$ & 15.09 & \pm 0.00 \\
\hline
\end{tabular}

a spectrum very similar to ours. Very recently, when the original version of this paper was already submitted for publication, Banerjee et al (2004, hereafter BVA04) presented infrared spectra obtained in September 2003 and April 2004. They have detected a water-ice absorption band and a $\mathrm{CO}$ band in emission. Thus V4332 Sgr is now an unusual emission-line object that deserves detailed observations. In this paper we present our observations and analyse the available data on the evolution of V4332 Sgr since its eruption in 1994.

\section{Observations}

The observations of V4332 Sgr were made at the South African Astronomical Observatory (SAAO). The spectra were obtained on 3 April 2003 with the grating spectrograph with SITe CCD mounted at the Cassegrain focus, $f / 18$, of the 1.9-m telescope. The total duration of the exposure was $900 \mathrm{~s}$ with the spectral coverage 3500-7400 $\AA$ and an average resolution of 1000 using grating No. 7 (300 lines/mm). The selected slit width was $300 \mu \mathrm{m}$ giving 1".8 projected on sky, comparable to seeing conditions during the observations. The $F W H M$ of the night sky lines was $5.6 \AA$. Two standard stars, CD-32 9927 and EG 274 , were observed during the night. This allowed us to calibrate the spectrum in flux units while the wavelength calibration was secured by observing CuAr lamp spectra.

The standard reduction procedures were applied to the spectra with the MIDAS ${ }^{1}$ longslit package. These included bias subtraction, flat-field correction, atmospheric extinction correction, wavelength and flux calibration.

Cousins $B V R_{\mathrm{c}} I_{\mathrm{c}}$ photometry was obtained with the $1.0-\mathrm{m}$ telescope and the $1 \mathrm{k} \times 1 \mathrm{k}$ STE4 CCD camera on 21 May 2003. The frames were bias-subtracted, overscan-trimmed and flatfielded using the CCDPROC package within IRAF. Target observations were calibrated against Landoldt standard stars. The results are given in Table 1 . Note that the errors given in the table are based only on the photon noise, they do not take into account external sources such as the calibration. Thus the true errors are probably larger than those quoted in the table.

The results in Table 1 can be compared to those obtained by BA04 on 29 September 2003. Apart from the $B$ magnitude, which is uncertain in both measurements and differs by 0.4 , the other magnitudes are consistent within $0.03-0.11$. Thus we can conclude that the object did not evolve significantly during the 4 month period in 2003 .

${ }^{1}$ MIDAS is developed and maintained by the European Southern Observatory.

\section{Spectrum}

The resultant spectrum of V4332 Sgr obtained on 3 April 2003 is presented in Fig. 1. The observational noise becomes excessive shortward of $\sim 4500 \AA$ where the spectrum is faint so that section is not shown in Fig. 1. For the remaining part a continuum level has been fitted by the median filtering of the spectrum and the observational noise has been derived by calculating the rms of the deviations from this continuum, excluding the apparent emission features. The calculated average noise is $0.19 \times 10^{-15} \mathrm{erg} \mathrm{cm}^{-2} \mathrm{~s}^{-1} \AA^{-1}$ and as a result the signal to noise ratio $(\mathrm{S} / \mathrm{N})$ for the detected continuum changes from only 1 to $\sim 5$ at the longest wavelenghs. However for the emission lines the $\mathrm{S} / \mathrm{N}$ can be substantially higher. Our spectrum can be compared to that of BA04. Note, however, that our wavelength range does not extend far enough into the red to register the prominent lines of KI.

As Fig. 1 shows, the spectrum is dominated by prominent emission features. This emission-line spectrum is, however, unusual and does not resemble any typical emission-line objects, such as planetary nebulae, symbiotic stars or T Tauri stars. Its is also significantly different from that displayed by V4332 Sgr during the 1994 eruption (MWT99) when the emission-line spectrum was dominated by the Balmer series. To some extent the present spectrum of V4332 Sgr can be compared to those of pecular giants, such as U Equ (e.g. Barnbaum et al. 1996) or VY CMa (e.g. Herbig 1974).

The strongest emission features in the spectrum of V4332 Sgr are identified in Fig. 1. Table 2 presents details of the emission lines, i.e. measured wavelengths (in $\AA$ ), line fluxes (in $10^{-15} \mathrm{erg} \mathrm{cm}^{-2} \mathrm{~s}^{-1}$ ) with errors in parenthesis, full width half maxima (in $\AA$ ), equivalent widths (in $\AA$ ) and the proposed identifications. For all lines the flux, FWHM and $E W$ were derived by performing a Gaussian fit to the observed profile using the REWIA package (J. Borkowski; http://www.ncac.torun.pl/ jubork). The errors on the line fluxes mostly depend on the uncertain continuum level and have therefore been calculated in an aproximate way as a product of the half of the average noise level and a doubled FWHM of a given line.

The strongest emission feature in our spectrum of V4332 Sgr is due to the unresolved Na I D lines. We have also been able to identify emission lines from other neutral atoms, i.e. Fe I and Ca I (see below).

The most interesting aspect of the present spectrum of V4332 Sgr is that it displays molecular bands in emission. The strongest and most numerous features are due to TiO. We have identified the prominent emissions near $7100 \AA$ with three components of band (0-0) of the $\gamma$ system corresponding to the transitions between electronic levels $A^{3} \Phi-X^{3} \Delta$. We believe that the (1-1) band of the same system at 7100-7200 (not marked in Fig. 1) is also visible in our spectrum. Another prominent band of $\mathrm{TiO}$ is that of $(0-0)$ of the $\gamma^{\prime}$ system $\left(B^{3} \Pi-X^{3} \Delta\right)$ near $6200 \AA$. Some other emission features can also be identified with excited bands of the $\mathrm{TiO} \gamma$ system. Following Herbig (1974) we have identified the prominent emission features at 6040 and $6080 \AA$ with $\mathrm{ScO}$. AlO, which dominates the near-IR spectrum of BVA03, is also present in 


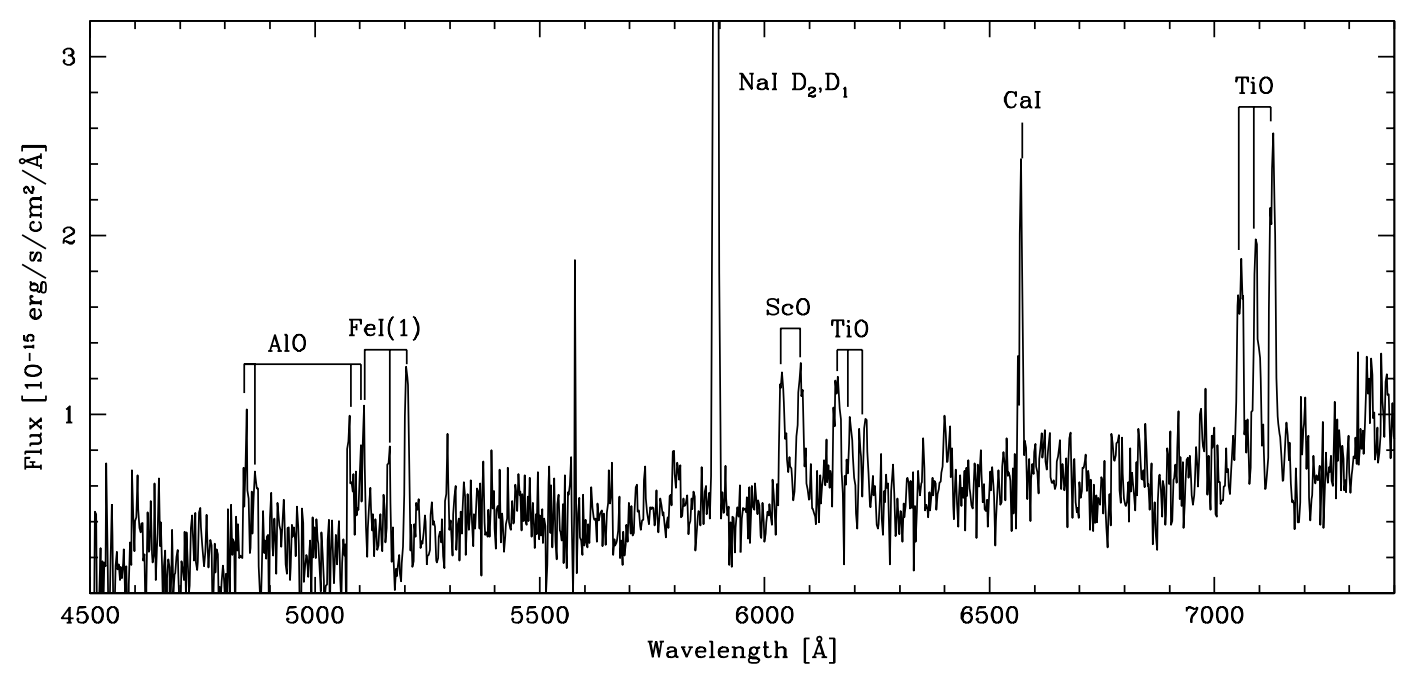

Fig. 1. The spectrum of V4332 Sgr obtained on 3 April 2003. The observed flux (in $10^{-15} \mathrm{erg} \mathrm{cm}^{-2} \mathrm{~s}^{-1} \AA^{-1}$ ) is shown versus the wavelength (in $\AA$ ). Due to the excessive observational noise, the spectrum below $\sim 4500 \AA$ is not shown. The sharp spike near $5580 \AA$ is due to imperfect sky subtraction. The labels identify the strongest emission features.

emission in our spectrum. Four bands of the $B^{2} \Sigma^{+}-X^{2} \Sigma^{+}$electronic transitions are clearly seen near $4850 \AA$ and $5100 \AA$.

The strong feature at $6569.8 \AA$ was initially attributed to $\mathrm{H}_{\alpha}$, but we subsequently identified it as the Ca I intercombination transition. $\mathrm{H}_{\alpha}$ was seen in the spectrum of V4332 Sgr during its 1994 eruption (MWT99), but at that time its mean position was $6558.7 \AA$. Since our position of the Na I D lines differs only by $1.3 \AA$ from that of MWT99, it is difficult to reconcile a difference of $11 \AA$ in the $\mathrm{H}_{\alpha}$ position. BA04 suggest that this feature might be due to $\mathrm{TiO} \gamma^{\prime}(0,1)$ as the wavelength matches well. In that case, other emissions originating from the same transition should be seen at $6596.3 \AA$ and $6629.0 \AA$ but they are not present. Furthermore, Table 2 shows that the $6569.8 \AA$ feature is significantly narrower than other molecular lines, suggesting that it is an atomic line. Given the very low excitation of the spectrum of V4332 Sgr, we consider Ca I $6572.8 \AA\left({ }^{1} \mathrm{~S}-{ }^{3} \mathrm{P}^{0}\right)$ as the most probable identification for the $6569.8 \AA$ feature.

As can be seen from Fig. 1, the spectrum shows a clear continuum rising toward longer wavelengths. The rather low signal-to-noise ratio does not allow unambiguous identification of absorption features. Yet a comparison of the observed spectrum with synthetic ones suggest an early M type.

An analysis of the strengths and widths of molecular bands allows us to estimate excitation conditions in the medium where the bands are produced. For the $\mathrm{TiO}$ bands we have used the data base described by Schwenke (1998) and distributed by Kurucz (1999). The observed widths of the emission peaks of the (0-0) band of the $\gamma$ system of TiO (7050-7130 $\AA$ ) has allowed us to estimate the rotational temperature, $T_{\text {rot }} \simeq 200 \mathrm{~K}$. On the other hand, the relative equivalent widths of these emission peaks point to the electronic temperature, $T_{\mathrm{el}} \gtrsim 500 \mathrm{~K}$. Following the analysis of Herbig (1974) and using the observed equivalent-width ratio of the $6079 \AA$ and $6036 \AA$ peaks we can estimate $T_{\text {rot }} \simeq 800 \mathrm{~K}$ for $\mathrm{ScO}$. It is worth noting that from numerous bands of $\mathrm{AlO}$ in the near-IR BVA03 estimated $T_{\text {vib }} \simeq 3000 \mathrm{~K}$ and $T_{\text {rot }} \simeq 300 \mathrm{~K}$. On the other hand, from the water ice feature at $3.05 \mu \mathrm{m}$ BVA04 have estimated a temperature of $30-50 \mathrm{~K}$, while their $\mathrm{CO}$ band analysis has suggested $300-400 \mathrm{~K}$.

\section{Analysis of the photometric data}

Using the available photometric data we have attempted to analyse the evolution of V4332 Sgr since its outburst in 1994. The method is the same as that used in Tylenda (2005) in the analysis of V838 Mon. Basically it fits standard colours from a sequence of spectral types to the photometric data. From the best fit, obtained using the least squares method, one derives the effective temperature, $T_{\text {eff }}$, and the angular stellar radius, $\theta$. This procedure requires the value of interstellar extinction to be known. Finally, if the distance to the object is known, the linear stellar radius, $R$, and the stellar luminosity, $L$, can then be calculated.

\subsection{Interstellar extinction}

The interstellar extinction to V4332 Sgr has been determined by MWT99. Comparing the photometric colours with the spectral classification from their spectra obtained in March 1994 they have found $E_{B-V}=0.32 \pm 0.02$. However, the values of $E_{V-R}$ and $E_{V-I}$ they obtained were significantly bluer, even negative in most instances (see their Fig. 6). This troublesome, at first sight, inconsistency can, however, be easily explained. The intrinsic colours which MWT99 compared with the photometric observations were in the Johnson (1966) system while the observations of Gilmore were done in the Cousins $\left(R_{\mathrm{c}}\right.$ and $\left.I_{\mathrm{c}}\right)$ system (Gilmore, private communication). Indeed, in Sects. 4.3 and 4.4 , there is a good agreement between the spectral types of MWT99 and the spectral types resulting from our fits to the $B V R_{\mathrm{c}} I_{\mathrm{c}}$ magnitudes if $E_{B-V}=$ 0.32 is assumed. We can also conclude that the uncertainty of this value is not greater than 0.1 . For $E_{B-V}$ beyond this range our spectral classes from the photomertic data become 
Table 2. Emission line spectrum of V4332 Sgr observed on 3 April 2003. ( $\lambda_{\text {obs }}, F W H M$ and $E W$ are in $\AA$. Flux is in $10^{-15} \mathrm{erg} \mathrm{cm}^{-2} \mathrm{~s}^{-1}$. Identification gives $\lambda_{\text {lab }}(\AA)$, element (molecule) and transition (for molecular transitions $\left(v^{\prime}-v^{\prime \prime}\right)$ means the upper and lower vibrational levels).)

\begin{tabular}{cccll}
\hline \hline \multicolumn{1}{c}{$\lambda_{\text {obs }}$} & Flux & $F W H M$ & $E W$ & Identification \\
\hline 4606.7 & $7.6( \pm 3.3)$ & 17 & 82 & \\
4846.9 & $7.8( \pm 2.0)$ & 11 & 52 & $4842.3 \mathrm{AlO} B^{2} \Sigma^{+}-X^{2} \Sigma^{+}(0-0)$ \\
4866.7 & $6.8( \pm 2.4)$ & 12 & 44 & $4866.4 \mathrm{AlO} B^{2} \Sigma^{+}-X^{2} \Sigma^{+}(1-1)$ \\
5078.1 & $8.4( \pm 2.3)$ & 12 & 41 & $5079.4 \mathrm{AlO} B^{2} \Sigma^{+}-X^{2} \Sigma^{+}(0-1)$ \\
5105.9 & $9.4( \pm 2.6)$ & 14 & 44 & $5102.1 \mathrm{AlO} B^{2} \Sigma^{+}-X^{2} \Sigma^{+}(1-2)$ \\
& & & & $5110.4 \mathrm{FeI}(1)$ \\
5123.1 & $3.3( \pm 1.7)$ & 9.2 & 15 & $5123.3 \mathrm{AlO} B^{2} \Sigma^{+}-X^{2} \Sigma^{+}(2-3)$ \\
5164.3 & $4.8( \pm 1.4)$ & 7.4 & 21 & $5166.3 \mathrm{FeI}(1)$ \\
& & & & $5168.9 \mathrm{FeI}(1)$ \\
5204.3 & $10.8( \pm 1.7)$ & 9.2 & 46 & $5204.6 \mathrm{FeI}(1)$ \\
5294.4 & $3.8( \pm 1.2)$ & 6.3 & 15 & \\
5802.2 & $7.4( \pm 3.9)$ & 21 & 19 & \\
5891.0 & $64.5( \pm 2.2)$ & 12 & 155 & $5889.9 \mathrm{NaI}$ \\
& & & & $5895.9 \mathrm{NaI}$ \\
6039.6 & $12.9( \pm 3.0)$ & 16 & 29 & $6036.1 \mathrm{ScO} A^{2} \Pi-X^{2} \Sigma(0-0)$ \\
6079.5 & $14.2( \pm 3.3)$ & 17 & 31 & $6079.2 \mathrm{ScO} A^{2} \Pi-X^{2} \Sigma(0-0)$ \\
6137.3 & $5.5( \pm 3.8)$ & 20 & 12 & \\
6161.2 & $12.4( \pm 2.9)$ & 15 & 26 & $6161.6 \mathrm{TiO} \gamma^{\prime}(0-0)$ \\
6190.7 & $6.5( \pm 2.4)$ & 13 & 14 & $6185.6 \mathrm{TiO} \gamma^{\prime}(0-0)$ \\
6221.6 & $8.3( \pm 3.6)$ & 19 & 17 & $6216.8 \mathrm{TiO} \gamma^{\prime}(0-0)$ \\
6403.5 & $9.0( \pm 4.1)$ & 22 & 18 & \\
6569.8 & $15.7( \pm 1.6)$ & 8.4 & 32 & $6572.8 \mathrm{CaI}\left({ }^{1} \mathrm{~S}-{ }^{3} \mathrm{P}^{0}\right)$ \\
6780.3 & $6.8( \pm 3.4)$ & 18 & 14 & $6781.3 \mathrm{TiO} \gamma(2-1)(?)$ \\
6840.3 & $9.3( \pm 6.5)$ & 34 & 19 & $6849.9 \mathrm{TiO} \gamma(3-2)(?)$ \\
& & & & $6814.7 \mathrm{TiO} \gamma(3-2)(?)$ \\
6974.8 & $10.2( \pm 4.0)$ & 21 & 21 & \\
7057.5 & $26.3( \pm 3.8)$ & 20 & 52 & $7054.3 \mathrm{TiO} \gamma(0-0)$ \\
7093.5 & $24.1( \pm 3.3)$ & 17 & 47 & $7087.7 \mathrm{TiO} \gamma(0-0)$ \\
7129.4 & $30.4( \pm 2.7)$ & 14 & 57 & $7125.5 \mathrm{TiO} \gamma(0-0)$ \\
& & & & $7124.9 \mathrm{TiO} \gamma(1-1)$ \\
7160.9 & $4.7( \pm 2.2)$ & 11 & 8.6 & $7158.5 \mathrm{TiO} \gamma(1-1)$ \\
7199.4 & $5.5( \pm 2.4)$ & 13 & 9.5 & $7197.2 \mathrm{TiO} \gamma(1-1)$ \\
& & & & $7197.2 \mathrm{TiO} \gamma(2-2)$ \\
7271.2 & $3.2( \pm 3.0)$ & 16 & 4.8 & $7270.4 \mathrm{TiO} \gamma(2-2)$ \\
7343.2 & $5.7( \pm 2.9)$ & 16 & 6.6 & \\
\hline & & & & \\
& & &
\end{tabular}

systematically discrepant from those of MWT99. In particular, from the fits done to the photometric data on 4-5 March (these data have been used by MWT99 to compare with their spectrum on 4.5 March and to derive $E_{B-V}=0.32$ ) assuming $E_{B-V}=0.20$ we have obtained K4.5-6.5 while for $E_{B-V}=0.45$ the result is $\mathrm{K} 2.0-3.0$. This can be compared to K3-4 obtained by MWT99 from their spectrum on 4.5 March.

In the present paper we assume $E_{B-V}=0.32$. The uncertainty in $E_{B-V}$ introduces uncertainties in the effective temperatures and the angular stellar radius derived from the fits discussed below. This errors can next propagate to the values of the linear stellar radius and luminosity. We have found that an error of 0.1 in $E_{B-V}$ affects the effective temperature and the angular stellar radius by at most $5 \%$. Thus in most cases this source of uncertainties is small compared to that due to the reference spectra (giants or supergiants) used in the fitting procedure (see Table 3 ). The resulting error in luminosity is larger,
Table 3. Evolution of V4332 Sgr in 1994-2003.

\begin{tabular}{cllccc}
\hline \hline Date & Sp. type & $T_{\text {eff }}$ & $-\log \theta$ & $R / R_{\odot}$ & $L / L_{\odot}$ \\
\hline 27.7 Feb.'94 & K0.2 I & 4400. & 8.85 & 114. & 4360. \\
& K1.7 III & 4470. & 8.86 & 110. & 4380. \\
3.7 Mar.'94 & K3.3 I & 4050. & 8.75 & 142. & 4880. \\
& K3.6 III & 4070. & 8.75 & 141. & 4950. \\
4.5 Mar.'94 & K3-4 III-I & 4400. & M. & & \\
4.7 Mar.'94 & K4.0 I & 3950. & 8.73 & 150. & 4910. \\
& K4.2 III & 3970. & 8.73 & 149. & 4980. \\
7.7 Mar.'94 & M1.1 I & 3640. & 8.75 & 141. & 2790. \\
& M1.1 III & 3710. & 8.80 & 128. & 2790. \\
8.7 Mar.'94 & M2.4 I & 3340. & 8.71 & 154. & 2690. \\
& M2.7 III & 3560. & 8.78 & 133. & 2550. \\
9.5 Mar.'94 & M3 III-I & 3800. & M. & & \\
10.7 Mar.'94 & M3.6 I & 3070. & 8.67 & 169. & 2290. \\
& M4.1 III & 3420. & 8.82 & 119. & 1760. \\
11.4 Mar.'94 & M5-6 III-I & 3100. & M. & & \\
& M3.8 I & 3040. & 8.89 & 102. & 790. \\
& M4.1 III & 3420. & 9.08 & 66. & 540. \\
20.4 Mar.'94 & M6-7 III-II & 2600. & M. & & \\
5-6 Jun.'94 & M8-9 III & 2300. & M. & & \\
& M9.0 I & 2120. & 8.97 & 86. & 135. \\
& M8.0 III & 3050. & 9.87 & 10.7 & 8.9 \\
18 May '98 & & & 9.96 & 8.7 & \\
& & & 10.06 & 6.9 & \\
11 Sep.'99 & M3.9 I & 3000. & 9.88 & 10.6 & 8.2 \\
& M4.4 III & 3390. & 10.06 & 6.9 & 5.6 \\
May-Sept.'93 & M2.7 I & 3280. & 10.13 & 5.9 & 3.6 \\
& M2.9 III & 3540. & 10.22 & 4.8 & 3.2 \\
\hline
\end{tabular}

i.e. $15-20 \%$, but even larger uncertainties are expected to result from the distance uncertainty (see Sect. 4.2).

\subsection{Progenitor and distance}

MWT99 have estimated that the distance to V4332 Sgr might be $\sim 300$ pc assuming that the object was a K giant at maximum. They have however concluded that this procedure did not lead them to consistent results if later observations are considered.

Skiff (1994), using the POSS prints, identified a possible progenitor of V4332 Sgr with $B=18$ and $R=16$. Using the ALADIN service we have searched for brightness estimates of the V4332 Sgr progenitor in archival surveys. We have found data in three catalogues. The SuperCOSMOS catalogue for a star at the position of V4332 Sgr gives $B=18.66$, $R=16.72$ and $I=15.89$. In the USNO-B1.0 catalogue the figures are $B=18.20, R=16.48$ and $I=15.69^{2}$. Finally in the USNO-A2.0 catalogue one finds $B=17.9$ and $R=16.4$. The results are fairly consistent although there are systematic differences between the values in the three catalogues. In all the bands the object is faintest in SuperCOSMOS while brightest in USNO-A2.0. The colours are more consistent. This is not

\footnotetext{
2 Both, SuperCOSMOS and USNO-B1.0 give second $R$ magnitudes for the V4332 Sgr progenitor, which are 14.96 and 14.38 , respectively. From the $R$ magnitudes of three nearby stars in these catalogues which in the red images are of similar brightness as the V4332 Sgr progenitor it can be safely concluded that these second magnitudes are significantly too bright.
} 
surprising as in authomatic measurements from survey plates or frames systematic errors are usually most important.

With $E_{B-V} \simeq 0.32$ (see Sect. 4.1) the colours from the above measurements imply an effective temperature of $5000-6000 \mathrm{~K}$. For this temperature range, the most reasonable assumption is that V4332 Sgr was a main sequence star prior to the eruption. Adopting intrinsic colours and absolute magnitudes for main sequence from Drilling \& Landoldt (2000) as well as assuming $E_{B-V}=0.32$ and the standard extinction curve the magnitudes from SuperCOSMOS can be best fitted with a $\mathrm{K} 0 \mathrm{~V}$ star at a distance of $1.35 \mathrm{kpc}$. The same procedure applied to the results from USNO-B1.0 gives a G6 V type and a distance of $1.58 \mathrm{kpc}$ while using the magnitudes from USNO-A2.0 one gets F8 V and $2.6 \mathrm{kpc}$.

If one assumes that before eruption V4332 Sgr was a giant rather than a main sequence star, then the observed colours of the progenitor would imply a spectral type of G5-F8 III and $M_{V} \simeq+1.0$ (Schmidt-Kaler 1982). Then the distance would increase to $\sim 10-13 \mathrm{kpc}$. This solution seems, however, to be unlikely given the position of V4332 Sgr in the sky, $l=13^{\circ} .63$ $b=-9^{\circ} .40$, and the observed reddening, $E_{B-V} \simeq 0.32$. Several planetary nebulae near this position, i.e. PNG 11.3-09.4, 12.5$09.8,13.8-07.9,13.7-10.6,14.2-07.3$, have $E_{B-V}$ in the range 0.37-0.52 (Tylenda et al. 1992), yet their distances are estimated to be within 3-8.5 kpc (Acker et al. 1992).

In the present paper we assume that V4332 Sgr was a solar type star before eruption and that the object is at a distance of $\sim 1.8 \mathrm{kpc}$ (mean value from the above estimates adopting main sequence). It is however clear from the above that this conclusion is subject to significant uncertainties. The distance value is probably uncertain to $50 \%$ or so introducing corresponding uncertainties in the values of the stellar radius and luminosity derived in Sect. 4.4.

\subsection{Observational data and fitting them with standard spectra}

During the 1994 eruption, the only multicolour $\left(U B V R_{\mathrm{c}} I_{\mathrm{c}}\right)$ photometry of V4332 Sgr was done by Gilmore (1994) and the data cover the period between 27 February and 11 March. MWT99 have estimated the BVR magnitudes from their spectra obtained on 11 March and 5-6 June 1994. From the published spectra in MWT99 we have made rough estimates of the I magnitude. Their Fig. 4 shows that on 11 March the flux in the $I$ band was $\sim 3.5$ times stronger than in the $R$ band which results in $R-I \simeq 2.2$. From Fig. 5 in MWT99 one can estimate that on 5-6 June the flux in the $I$ band was $\mathrm{F}_{\lambda} \simeq 3.5 \times 10^{-14} \mathrm{erg} \mathrm{cm}^{-2} \mathrm{~s}^{-1} \AA^{-1}$ giving $I \simeq 10.9$.

In the 2MASS survey the object was observed on 18 May 1998 and the results were: $J=12.10, H=11.60$ and $K=10.99$. V4332 Sgr was also measured in the DENIS survey on 11 September 1999 which gave $I_{\text {Gunn }}=14.37, J=12.46$ and $K_{\mathrm{s}}=10.66$.

In 2003, apart from our $B V R_{\mathrm{c}} I_{\mathrm{c}}$ photometry obtained on 21 May and summarized in Table 1, optical photometry was also done by BA04 on 29 September. Besides, JHK magnitudes were obtained by BVA03 on 19 June 2003, while LM

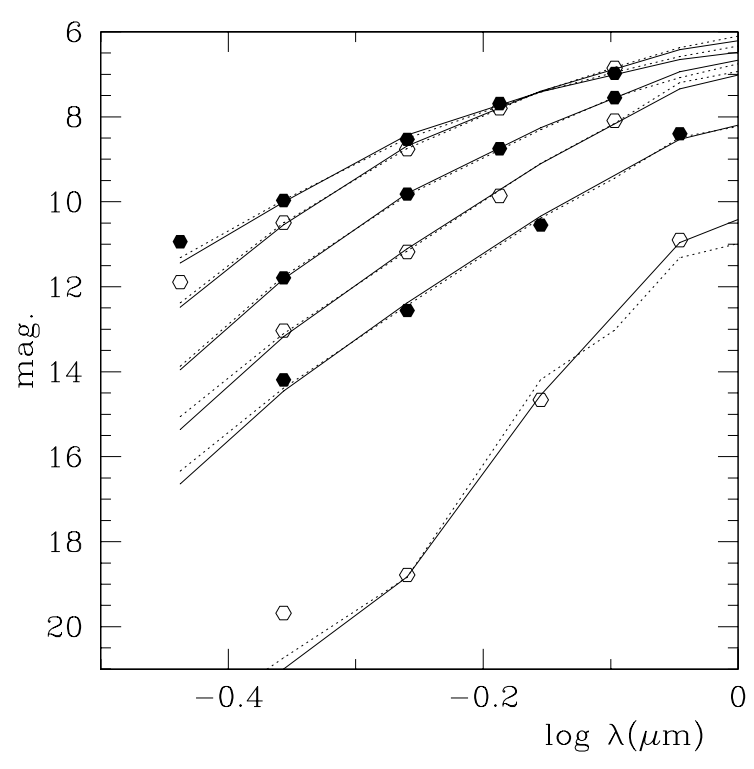

Fig. 2. Evolution of the spectrum (magnitude versus effective wavelength) of V4332 Sgr in 1994. Symbols show the observed magnitudes (taken from Gilmore 1994 and MWT99). Solid and dotted curves show the fitted standard supergiant and giant spectra, respectively (reddened using the standard extinction curve and $E_{B-V}=0.32$ ). From top to bottom the curves correspond to the observations made on 27.7 February, 4.7 March, 7.7 March, 10.7 March, 11.4 March and 5-6 June 1994, respectively.

magnitudes measured on 5 September 2003 can be found in BVA04. As discussed in Sect. 2, V4332 Sgr did not evolve significantly between May and September. Therefore we have combined all the data from 2003 into one set for the analysis discussed below. In particular, in the optical we have taken mean values from our results in Table 1 and those of BA04.

When fitting the observed magnitudes, we applied the standard intrinsic colours for the luminosity class III (giants) and I (supergiants). This is justified for the period of the 1994 eruption as the observed spectra of V4332 Sgr then resembled those of the III-I luminosity class (MWT99). Also, for the data obtained in 2003 we have found that the giant or supergiant spectra better fit the observations than the colours for main sequence stars. The intrinsic photometric colours have been taken from Schmidt-Kaler (1982 - UBV), Johnson (1966 - VRI for $\mathrm{G}$ and K types), Lee (1970 - VRI for M types), Bessell \& Brett (1988 - VI $I_{\mathrm{c}} J H K L M$ for giants) and Koornneef (1983 - VJHKLM for supergiants). The calibration of the effective temperature and the bolometric correction against the spectral type has been taken from Schmidt-Kaler (1982).

Figure 2 presents the best fits of the standard giant (dotted curves) and supergiant (solid curves) spectra to the photometric data obtained in 1994. Note that for clarity of the figure the spectra on 3.7 March and 8.7 March have not been displayed in Fig. 2.

The results of the fits, i.e. spectral types, effective temperatures and angular stellar radii, $\theta$ (in radians), are given in Table 3. For each date the results of the fits are given in two lines: first the results from fitting the supergiant (I) spectra, second those for - the giants (III). The lines marked with M in the 


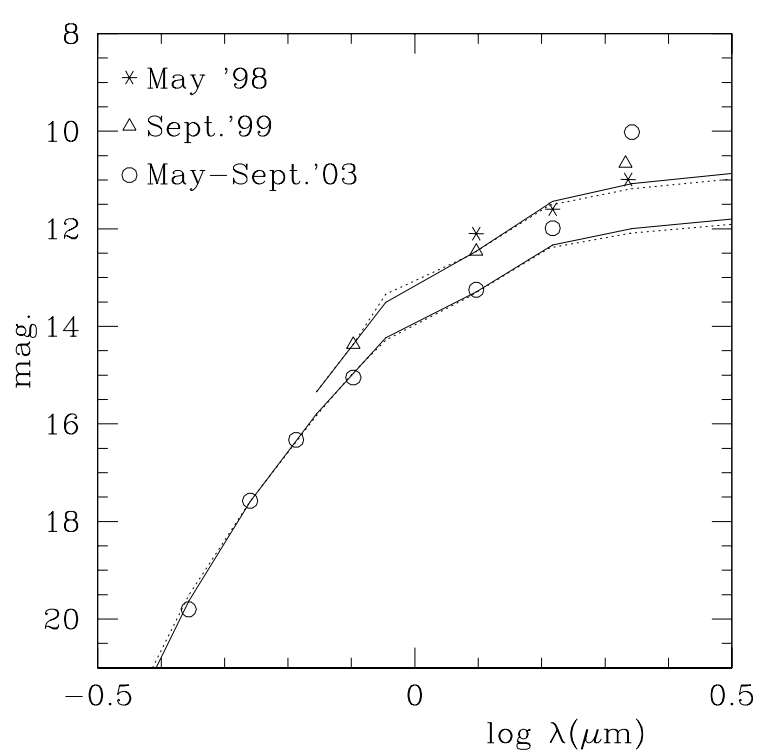

Fig. 3. The spectrum of V4332 Sgr in 1998, 1999 and 2003. Asterisks $-J H K$ in May 1998 from 2MASS. Triangles $-I_{\text {Gunn }} J K_{\mathrm{s}}$ in September 1999 from DENIS. Circles $-B V R_{\mathrm{c}} I_{\mathrm{c}} J H K$ on MaySeptember 2003 (see the text). Full and dotted curves - standard supergiant and giant (respectively) spectra fitted to the 1999 and 2003 data (for details see the text). Parameters of the fits are given in Table 3. The model spectra have been reddened with $E_{B-V}=0.32$.

fourth column give the results from MWT99, i.e. spectral type from classification and effective temperature from stellar atmosphere model analysis of their spectra.

Note that the $U$ magnitude, measured by Gilmore only for three initial dates, has not been taken into account in the fitting procedure as its inclusion degrads the fit quality. This is not suprising as the $U$ band is dominated by the Balmer continuum which is very sensitive to nonstandard phenomena such as departures from hydrostatic equilibrium, non-LTE and winds. As can be seen from Fig. 2, V4332 Sgr was systematically brighter by $\sim 0.5$ mag in the $U$ band when compared to the standard supergiants and giants.

Note also that when fitting the magnitudes derived from MWT99 for 5-6 June, the $B$ magnitude was not taken into account. It was presumably affected by strong emission lines, as can be seen from Fig. 5 in MWT99. The results obtained for this date are particularly uncertain and should be regarded with caution. They are based on rough estimates of the VRI magnitudes made from the spectral observations. Besides, judging from the estimated effective temperature the observations covered only the shortwavelength Wien's part of the spectrum. The bulk of the energy of the object was presumably emitted in the infrared where no measurements were made. Finally, our fits for this date result from extrapolation beyond the range of the standard spectra (the latest types for which intrinsic colours are available are: M 6-7 for giants and M 5-6 for supergiants).

Figure 3 shows the results of observations in 1998, 1999 and 2003. Asterisks indicate the 2MASS measurements obtained on 18 May 1998. Triangles show the DENIS results derived on 11 September 1999. Circles represent the spectrum observed in May-September 2003 (see above for the sources of the data).

First we discuss the observations obtained in 2003 as they cover the largest spectral range. The whole spectrum, i.e. all the circles in Fig. 3, cannot be fitted with a single standard spectrum. This can only be done for shorter wavelengths. In Fig. 3 we show the best fits of the supergiant (full curve) and giant (dotted curve) spectra for the $V R_{\mathrm{c}} I_{\mathrm{c}} J$ measurements. The parameters of the fits are given in Table 3 . The $B$ magnitude has not been taken into account in the fitting procedure because of its significant uncertainty. However, as can be seen from Fig. 3, it fits well the obtained spectra. In the long wavlength range the $H$ and, particularly, $K$ magnitudes show a clear excess compared to the spectra fitted in the shorter wavelengths. With the $L$ and $M$ magnitudes measured by BVA04 (not shown in Fig. 3) one can easily conclude that the source of this excess dominates the brightness of the object in the infrared. This infrared excess will be discussed in Sect. 6 .

The infrared observations displayed in Fig. 3 show that V4332 Sgr evolved systematically between 1998 and 2003. It was becoming fainter in $J$ (and $I$ ) but brighter in $K$. This can be interpreted as due to a gradual fading of the main object (seen in $J$ and $I$ ) and the increasing infrared excess in $K$. Therefore when fitting the standard spectra to the DENIS magnitudes obtained on 11 September 1999 (triangles in Fig. 3), expecting that $K$ may be affected by the IR excess, we considered only $I$ and $J$. Indeed, as can be seen from Fig. 3, the $K$ magnitude is $\sim 0.5 \mathrm{mag}$ above the fitted spectrum. Note, however, that if all the three $(I J K)$ measurements are taken into account the fits would be quite poor and $T_{\text {eff }}$ would lower by $\sim 100 \mathrm{~K}$ for class III and $\sim 200 \mathrm{~K}$ for class I compared to the values given in Table 3 .

No fit to the 2MASS data on 18 May 1998 (asterisks) is shown in Fig. 3. The spectral range of the data is quite narrow and hence any $T_{\text {eff }}$ between $\sim 3000 \mathrm{~K}$ and $\sim 4500 \mathrm{~K}$ could be considered to satisfactorily fit the data. Fortunately for this wide $T_{\text {eff }}$ range, $\log \theta$ of the fits varies by less than 0.20 . The mean values of $\log \theta$ from these fits are given in Table 3 .

\subsection{Evolution of the object}

The last two columns in Table 3 show the stellar radius and luminosity (given in solar units) of V4332 Sgr calculated from the angular radius $(\theta)$ and the effective temperature $\left(T_{\text {eff }}\right)$ assuming a distance of $1.8 \mathrm{kpc}$.

Figure 4 displays the evolution of principal parameters of V4332 Sgr taken from Table 3. The time is in days since 24 February 1994, i.e. since the discovery of V4332 Sgr in eruption. The effective temperature is in units of $10^{3} \mathrm{~K}$, while the stellar radius and luminosity are in solar units. Open symbols show the results from fitting the standard supergiant (I) spectra to the observations. The same but using the giant (III) spectra is presented with the full points. Asterisks in panel a denote the effective temperatures derived by MWT99 from fitting stellar atmosphere models to their spectra.

Let us first discuss the spectral types obtained from our analysis. As can be seen from Table 3 no matter which 

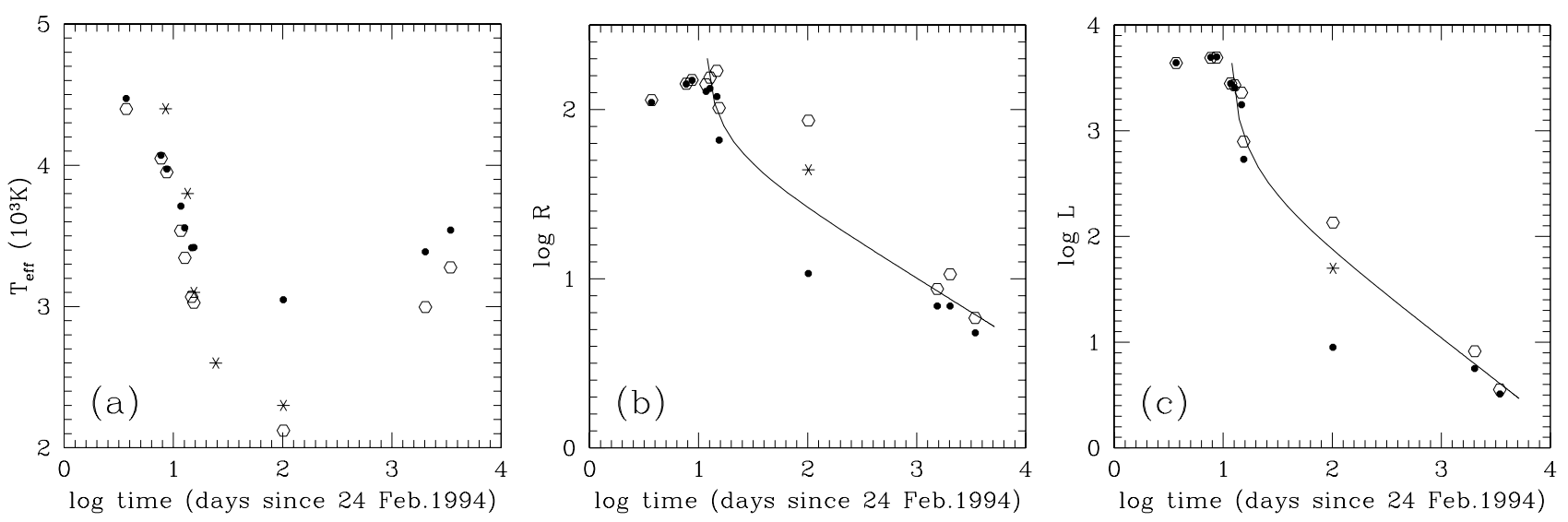

Fig. 4. Evolution of V4332 Sgr with time. Time (displayed in log scale) is in days counted since the discovery of the object in eruption (24 Feb. 1994). Left a) - effective temperature in $10^{3} \mathrm{~K}$. Centre b) - logarithm of radius in $R_{\odot}$. Right $\mathbf{c}$ ) - logarithm of luminosity in $L_{\odot}$. Open symbols - results from fitting the supergiant (I) spectra. Full points - results from fitting the giant (III) spectra. Asterisks in a) - effective temperature from MWT99. Asterisks in b) and c) - estimates on 5-6 June 1994 based on the results of MWT99 as discribed in the text. Full curves in $\mathbf{b})$ and $\mathbf{c}$ ) - results of a model discribed in Sect. 5.

luminosity class of the standard spectra (I or III) is used, the resultant spectral types are always very similar. Moreover, our spectral classes are also very close to those obtained by MWT99 from the classification of their spectra. This shows that the general approach adopted in our analysis of the photometric data is consistent and reliable.

The consistency of the effective temperatures derived in the different ways is not as good as that of the spectral types. As can be seen from Table 3 and Fig. 4a initially, when the object was of the K type, both kinds of standard spectra give practically the same values of $T_{\text {eff }}$. Later, when the object was becoming cooler, an increasing discrepancy appears in the sense that $T_{\text {eff }}$ obtained using the supergiant spectra is systematically lower than that from using the giant spectra. The reason lies in the calibrations of $T_{\mathrm{eff}}$ versus spectral type used in our study (Schmidt-Kaler 1982). For the same spectral type, the value of $T_{\text {eff }}$ for supergiants is systematically lower than that for the giants but for the $\mathrm{G}, \mathrm{K}$ types the difference is small. For the $\mathrm{M}$ types the discrepancy increases and becomes as large as $530 \mathrm{~K}$ at M5. This has obvious consequences for the derived effective radii and luminosities: the use of the supergiant standard spectra usually results in larger radii and luminosities.

Comparing our results with those obtained by MWT99, one sees that on March 4 and 9 their $T_{\text {eff }}$ is systematically higher. This agrees with what was noted in MWT99, namely that the value of $T_{\text {eff }}$ they obtained from the stellar model analysis was too high for the observed spectral type. MWT99 ascribed this discrepancy to the narrow observed spectral range. On the later dates the spectral types and $T_{\text {eff }}$ derived in MWT99 are consistent and, as can be seen from Fig. 4a, their $T_{\text {eff follows quite }}$ closely our values obtained using the supergiant spectra.

Note that, as discussed in Sect. 4.3, our results obtained from the data on 5-6 June 1994 (symbols at $\log t \simeq 2.0$ in Fig. 4) are subject to significant uncertainty. They are based on uncertain observational estimates done only in the Wien's part of the spectrum, as well as, on the extrapolated standard spectra. Due to the increasing discrepancy in the effective temperature between giants and supergiants for the late M types, discussed above, the difference between our estimates of $T_{\text {eff }}$ is as large as $900 \mathrm{~K}$. This resulted in large differences in the stellar radius and luminosity seen in Table 3 and Fig. 4. MWT99, from their model atmosphere analysis, estimated that by 5-6 June the object had faded by a factor of 100 in luminosity compared to the beginning of March. Adopting our determinations of $\sim 5000 L_{\odot}$ for the beginning of March this results in an estimate of $\sim 50 L_{\odot}$ and, assuming $T_{\text {eff }}=2300 \mathrm{~K}$ (from MWT99), an effective radius of $\sim 44 R_{\odot}$ on 5-6 June. These estimates are shown with asterisks in Figs. 4b,c.

As can be seen from Table 3 and Fig. 4c during $\sim 10$ days after its discovery, V4332 Sgr sustained a fairly constant luminosity, the star being $\sim 5000$ times brighter than before the eruption. The radius was then $\sim 140$ times larger than that of the progenitor, possibly slowly expanding with time. Near 5 March, the stellar luminosity declined rapidly and during $\sim 6$ days the object faded by a factor $\sim 7$.

Concerning the effective radius we can only state, given the uncertainties in our results, that the radius was not changing significantly till 10 March. It seems, however, that a significant shrinkage of the radius occurred near 11 March. The initial luminosity decline was thus primarily due to the decline in the effective temperature.

The subsequent fading involved the decline of all the stellar parameters, i.e. $L, T_{\text {eff }}$ and $R$. At a certain time $T_{\text {eff }}$ reached a minimum but the available data do not permit an estimate of when it occurred and how deep this minimum was. From Fig. 4 it can be inferred that this occured near June 1994 or later. The lowest observed $T_{\text {eff }}$ was reached on 5-6 June 1994, i.e. some 90 days after the luminosity started declining. During this time interval, the object dropped in luminosity by factor at least $\sim 40$. According to MWT99, this luminosity drop was of factor 100 and the object became as cool as $2300 \mathrm{~K}$.

The decline in luminosity and radius has continued, although at a decreasing rate. This has been followed by a slow rise in the effective temperature and at present the object is significantly hotter than in June 1994. Comparing the results obtained in 2003 with the estimates made for the progenitor (see 
Sect. 4.2), we can conclude that V4332 Sgr is now larger and more luminous, but cooler than before the eruption, so it has not yet returned to its pre-outburst state.

\section{Gravitational contraction in the decline}

The fading of V4332 Sgr can be consistently interpreted if one assumes that the energy source, that caused V4332 Sgr to erupt to $\sim 140 R_{\odot}$, suddenly disappeared. This resulted in a gravitational contraction of the inflated envelope and a release of its gravitational energy. To show this, let us assume that the inflated envelope has a density distribution of $\rho \sim r^{-5 / 2}$ (following Soker \& Tylenda 2003). Then, assuming that the envelope mass, $M_{\mathrm{e}}$, is small compared to the star mass, $M_{\star}$, it is straightfoward to show that the gravitational energy of the envelope is

$E_{\mathrm{g}}=-\frac{G M_{\star} M_{\mathrm{e}}}{\left(R_{\star} R\right)^{1 / 2}}$,

where $G$ is the gravitational constant, $R_{\star}$ is the radius of the unperturbed stellar layers (assumed to be the inner radius of the envelope) and $R$ is the outer radius of the envelope. If the radiated luminosity, $L$, is due to gravitational contraction of the envelope, one gets

$L=-\frac{\mathrm{d} E_{\mathrm{g}}}{\mathrm{d} t}=\frac{G M_{\star} M_{\mathrm{e}}}{2 R_{\star}^{1 / 2}} \frac{1}{R^{3 / 2}} \frac{\mathrm{d} R}{\mathrm{~d} t}$.

On the other hand

$L=4 \pi R^{2} \sigma T_{\mathrm{eff}}^{4}$.

Combining Eqs. (2) with (3) gives

$\frac{\mathrm{d} R}{R^{7 / 2}}=-\frac{8 \pi R_{\star}^{1 / 2} \sigma T_{\mathrm{eff}}^{4}}{G M_{\star} M_{\mathrm{e}}} \mathrm{d} t$.

Assuming that $T_{\text {eff }}$ is constant (which is obviously a very crude approximation), Eq. (4) can be integrated giving

$\frac{1}{R^{5 / 2}}=\frac{20 \pi R_{\star}^{1 / 2} \sigma T_{\mathrm{eff}}^{4}}{G M_{\star} M_{\mathrm{e}}} t+\frac{1}{R_{0}^{5 / 2}}$,

where $R_{0}$ is the initial envelope radius assumed to start contracting at $t=0$.

The solid curves in Fig. 4b,c shows the evolution of a gravitationally contracting envelope calculated from Eq. (5). The solar values for the stellar paramaters, i.e. $M_{\star}=1.0 M_{\odot}$ and $R_{\star}=1.0 R_{\odot}$, have been assumed, as well as, $T_{\text {eff }}=3300 \mathrm{~K}$ as a typical effective temperature during the decline. The mass of the envelope have been chosen to fit the observed evolution and in the case shown in Figs. $4 \mathrm{~b}, \mathrm{c}, M_{\mathrm{e}}$ is $1.5 \times 10^{-5} M_{\odot}$. We note that values of $M_{\mathrm{e}}$ differing by less than a factor 3 , i.e. being within $0.5-5.0 \times 10^{-5} M_{\odot}$, can be considered as reasonably reproducing the observations. Given the crudness of our approach and uncertainties in the observational determinations of $R$, we can conclude that pure gravitational contraction of the envelope inflated during the eruption can satisfatorily explain the observed decline of V4332 Sgr. The mass of the envelope is small, of order $10^{-5} M_{\odot}$. Note that the total mass involved in the eruption was probably significantly larger than $M_{\mathrm{e}}$. Some mass was probably lost from the object and/or is circulating the object in a disc-like structure (see Sect. 6).

\section{Infrared excess in 2003}

One of the most important features in the spectrum of V4332 Sgr in 2003 is the infrared excess clearly seen in the $H$ and $K$ bands in Fig. 3. As discussed in Sect. 4.3, no such excess is necessary to explain the data in 1998. The 2MASS magnitudes can be well accounted for by a cool star somewhat brighter then the stellar component seen in 2003. In September 1999 the $K$ band was probably affected by an excess, although its magnitude was certainly significantly smaller than that in 2003 .

In order to discuss the origin of this IR excess, we have attempted to fit the observations in 2003 with multicomponent spectra. Symbols in Fig. 5 show the spectrum in May-September 2003 (see Sect. 4.3 for the sources of the data). The full curve shows a fit of the data with a two component spectrum: a standard supergiant spectrum of $T_{\text {eff }}=3280 \mathrm{~K}$ (M2.7 I - the same as in Fig. 3) and a blackbody distribution with $T_{\mathrm{bb}}=750 \mathrm{~K}$. As can be seen from Fig. 5 the fit is very good. The angular radius of the blackbody component in Fig. 5 is $\log \theta=-8.28$, which at a distance of $1.8 \mathrm{kpc}$ gives an effective radius of $\sim 400 R_{\odot}$ and a luminosity of $\sim 50 L_{\odot}$.

Fits of the kind shown in Fig. 5 can be interpreted as evidence for a dusty envelope that reprocesses radiation absorbed from the central star. This interpretation is however very unlikely. The infrared component is $\sim 15$ times brighter than our estimate of the stellar luminosity in Table 3. This would imply that the central star is significantly more luminous and that what we observe in the optical is the stellar spectrum severely attenuated by dust. Then the central star, reddened by dust, would be much hotter than estimated from the photometry. Yet, as noted in Sect. 3, our spectrum suggests an early M type star, thus consistent with the photometric results. Besides, the large present luminosity and the presence of hot nearby dust would inevitably indicate that V4332 Sgr has recently experienced another outburst. With the effective radius of the blackbody component estimated above $\left(\sim 400 R_{\odot}\right)$ and adopting a rather lower limit of $10 \mathrm{~km} \mathrm{~s}^{-1}$ for the expansion velocity, one finds that dust would have been lost a year before May-September 2003. However, as discussed in Sect. 4.3, the infrared excess started developing in 1999. Besides, there is no other observational indication that the object recently suffered from a secondary outburst. On the contrary, as can be seen from Fig. 4, the object has been following the long term decline after the 1994 eruption. The lack of any significant evolution in the optical magnitudes between May and September 2003 is also an evidence for the long time scale evolution of the object in 2003.

BVA04 have recently considered that V4332 Sgr might be a young object surrounded by a protostellar circumstellar disc. This hypothesis has been invoked to explain the origin of the water-ice band observed in the infrared spectrum. It also allows BVA04 to suggest, following the ideas of Soker \& Tylenda (2003) and Retter \& Marom (2003), that the 1994 outburst of V4332 Sgr might have been due to infall of an inner planet due to tidal interaction with the disc. According to BVA04 the presently observed emission spectrum and the infrared excess, which appeared in 1999, might have been due to interaction of the 1994 ejecta with the circumstellar disc. However, an 


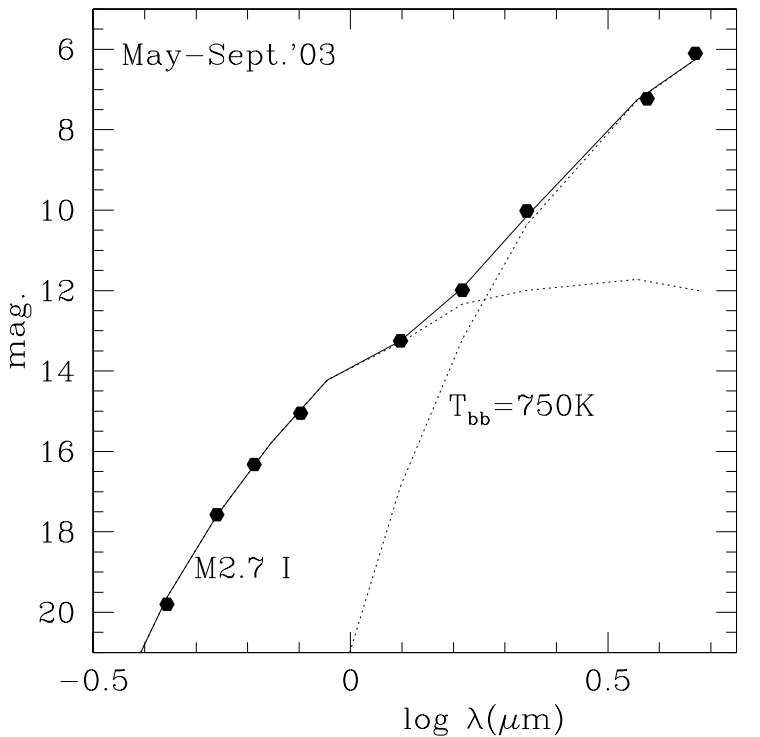

Fig. 5. The magnitudes observed in May-September 2003 (see Sect. 4.3 for the sources of the data) fitted with a two component spectrum: M 2.7 supergiant of $T_{\text {eff }}=3280 \mathrm{~K}$ and blackbody of $T=750 \mathrm{~K}$. Symbols - observed magnitudes. Dotted curves - individual component spectra. Full curve - sum of the two components.

interaction of this kind should have happen during the 1994 eruption or soon after it. Assuming an expansion velocity of $100 \mathrm{~km} \mathrm{~s}^{-1}$ (MWT99) the distance of $400 R_{\odot}$ is reached in a month. Thus it is not clear why the IR excess might have appeared $\sim 5$ years later and why it might have been brightening with time, as observed.

The idea of a protostellar disc can however be used to explain the increasing IR excess, although in a different way to that in BVA04. In the case of an inner planet and an external accretion disc, tidal forces transfer angular momentum from the orbital motion of the planet to the inner edge of the disc. As a result the inner edge of the disc is kept at a certain distance from the planet orbit, determined by a balance between the rate of the angular momentum gain from the planet and the rate in which the angular momentum is transported outward in the disc. When the planet gets accreted by the central star the eruption can disturb the disc, possibly destroying its inner regions. After the event the inner disc does not gain angular momentum any more. As a result the inner edge will be approaching the star and the inner disc will become hotter and hotter. Thus we can speculate that just after the 1994 eruption the inner disc in the V4332 Sgr system was far away, cold and thus radiating only in the far IR, while at present the inner disc regions are closer to the star, thus they are hotter and dominating the observed brightness in the KLM bands.

There are, however, observational facts which do not favour the hypothesis that V4332 Sgr is a young object. First, the position of V4332 Sgr in galactic coordinates is $l=13.63$, $b=-9.40$. According to catalogues of Sharpless (1959), Lynds (1965), Avedisova (2002) and Russeil (2003) there is no HII or star forming region closer than $5^{\circ}$ from the position of V4332 Sgr. Also from the CO map of Dame et al. (2001, Fig. 2) one can see that the object lies well outside any significant CO emission. Second, MWT99, from their high resolution spectra near $\mathrm{H}_{\alpha}$, have derived a radial velocity of V4332 Sgr to be $-180 \mathrm{~km} \mathrm{~s}^{-1}$. A similar value, i.e, $-160 \mathrm{~km} \mathrm{~s}^{-1}$, can be obtained from the observed positions of all the emission lines listed in their Table 3. This result has been derived from outburst spectra so interpretations other than the radial velocity of the object (although very unlikely) can be considered (see MWT99). However, the observed wavelengths of the $\mathrm{CaI}$ line in our Table 2 still gives a similar radial velocity, i.e $-140 \mathrm{~km} \mathrm{~s}^{-1}$. The Galactic rotation curve (see e.g. Brand \& Blitz 1993) predicts, for the position of V4332 Sgr and a distance of $1.8 \mathrm{kpc}, V_{\mathrm{LSR}}=+13 \mathrm{~km} \mathrm{~s}^{-1}$ which is equivalent to a heliocentric radial velocity of $\sim+3 \mathrm{~km} \mathrm{~s}^{-1}$. A CO radial velocity map of Dame at al. (2001, Fig. 3) shows, at the galactic longitude of V4332 Sgr, $V_{\mathrm{LSR}}$ between 0 and $+140 \mathrm{~km} \mathrm{~s}^{-1}$. Thus V4332 Sgr does not follow the Galactic rotation which is not expected to be the case for a young object.

Formation of a circumstellar disc is, however, also possible in the merger scenario proposed by Soker \& Tylenda (2003) in which a star accretes a less massive object. In this case, the inflated stellar envelope gains not only energy from the merger, but also angular momentum from the orbital motion. Note that if the merger event is provoked by an interaction with a fast moving object (as discussed above V4332 Sgr is probably moving with a velocity $\gtrsim 200 \mathrm{~km} \mathrm{~s}^{-1}$ relative to the objects rotating in the Galactic disc) the angular momentum accreted is likely to be much larger than that of the Keplerian motion near the star surface. During the outburst, i.e. when the envelope remains inflated to large radii, processes due to turbulent or convective motions, tidal effects, as well as magnetic fields are likely to transport angular momentum outward. When the merger process is more or less complete, the inflated envelope would tend to collapse but the angular momentum stored in the equatorial regions may prevent these regions from contracting significantly. They would form a ring-like or disc-like structure orbiting the central object. During the fading phase when the contracting central object is likely to rotate significantly faster than the matter orbiting at larger distances, magnetic fields and tidal forces can continue to transport angular momentum outward. With time, due to viscous processes, the ring-like structure would evolve toward an accretion disc and dissipate its energy.

Following the above discussion we have fitted the observations in 2003 with a composite spectrum of a central star and a standard blackbody accretion disc. The central star spectrum is the same as in Fig. 5. The distribution of the effective temperature of the disc is (see e.g. Eq. (5.43), in Frank et al. 2002)

$T(R)=T_{0}\left\{3\left(\frac{R_{0}}{R}\right)^{3}\left[1-\left(\frac{R_{0}}{R}\right)^{1 / 2}\right]\right\}^{1 / 4}$

where

$T_{0}=\left(\frac{G M_{*} \dot{M}}{8 \pi R_{0}^{3} \sigma}\right)^{1 / 4}$

$R_{0}$ is the inner radius of the disc while $\dot{M}$ is the accretion rate. Note that $T(R)$ attains a maximum value $0.642 T_{0}$ at 
$R=1.36 R_{0}$. Integrating the blackbody spectrum with $T(R)$ over the disc surface one gets the disc spectrum.

We do not show the final fit to the observations as it is very much the same as that in Fig. 5. It has been obtained for $T_{0}=1400 \mathrm{~K}$ and $\log \left(\theta_{0}\right)=-8.70$, where $\theta_{0}$ is the "observed" angular inner radius of the disc. Assuming a distance of $1.8 \mathrm{kpc}$ and $M_{*}=1 M_{\odot}$ this translates to $R_{0} \simeq 160 R_{\odot}$ and $\dot{M} \simeq 8.8 \times 10^{-4} M_{\odot} / \mathrm{yr}$. The total luminosity of the disc is then $\sim 87 L_{\odot}$. Note that $R_{0}$ is very close to the maximum radius of the star during the 1994 eruption (see Table 3). This suggests that if the disc is of protostellar origin its inner regions have probably been distroyed during the eruption, as discussed above.

Taking Eq. (5.69) in Frank et al. (2002) and assuming the viscosity parameter, $\alpha=0.01-0.1$, one gets, for the above values of $R_{0}$ and $\dot{M}$, the viscous time scale of $4-20$ years. Thus one can argue that in 1998-1999 the inner edge of the disc or ring-like structure was somewhat farther away and cooler than in 2003, the star was brighter so the relative contribution of the structure in the $H K$ bands was negligible.

\section{Discussion and conclusions}

There is no doubt that the eruption of V4332 Sgr observed in 1994 was a highly unusual, enigmatic and extremely interesting astrophysical event. This statement results not only from the observed behaviour of the object in 1994 but also from its present state marked by the unusual emission line spectrum and the mysterious infrared excess. It is most unfortunate that so little information is available regarding the evolution of V4332 Sgr during and after its 1994 eruption. The data is limited to the optical with just the six multicolour photometric observations of Gilmore (1994) covering 11 days and seven spectra of MWT99 obtained in a three month period. The lack of infrared data is particularly disappointing since the object was likely emitting much of its energy in this wavelength region.

The results of our photometric analysis in Sect. 4, supplemented with the results of MWT99, clearly show that the decline of V4332 Sgr following its 1994 eruption was marked by a decline in all principal parameters: luminosity, radius and effective temperature. In 2003 the object was $\sim 1500$ times less luminous than during the eruption but still remaining in the spectral range of the $\mathrm{M}$ type. As discussed in Soker \& Tylenda (2003), this type of evolution rules out the classical nova mechanism which could have worked for a hypothetical white dwarf companion to the progenitor. Note also that in June 1994 and May-September 2003, V4332 Sgr was significantly (1.0-1.5 mag.) fainter in the $B$ band than the progenitor (between these two dates there were no measurements in $B$ ). This implies that it was the progenitor which erupted in 1994 and not its hypothetical faint companion.

As discussed in Sect. 5, the decline of V4332 Sgr can be understood in terms of a pure gravitational contraction of the inflated stellar envelope. An event of this kind is expected to follow a sudden switch-off of the energy sources which have so far been working at the base of the envelope. This interpretation is well in line with the stellar merger scenario of Soker \& Tylenda (2003). When the merger phase is complete, the source of energy quickly drops and the inflated envelope has to contract under the gravity of the star. The considerations in Sect. 5 show that in order to explain the observed decline of V4332 Sgr over almost a decade, the mass of the inflated stellar envelope must be small, of order $10^{-5} M_{\odot}$.

The origin of the infrared excess dominating the observed KLM magnitudes in 2003 is unclear. When fitted with blackbody it implies a temperature of $\sim 750 \mathrm{~K}$ and a luminosity $\sim 15$ times higher than that of the central star. As discussed in Sect. 6 it is very unlikely that the excess is due to a dusty envelope reprocessing the radiation from the central star. We have considered that the IR radiation can originate from a circumstellar disc dissipating its gravitational energy via viscous processes. The disc can be either of protostellar origin, following suggestions of BVA04, or being a remnant storing angular momentum after the 1994 eruption. Both possibilities are in line with the merger scenario of Soker \& Tylenda (2003). The first one assumes that V4332 Sgr is a young object. This hypothesis is however inconsistent with the position of the object in Galaxy and, particularly, with its radial velocity significantly different from the Galactic rotation curve. Future observations of V4332 Sgr in the infrared, particularly in longer wavelengths might be crucial for understanding the nature of the object.

A lower limit to the mass of this object accreted in the merger scenario of Soker \& Tylenda (2003) can be obtained from the total energy emitted in the eruption. The luminosity of V4332 Sgr integrated since the discovery of the 1994 eruption till 2003 is $\sim 4.5 \times 10^{43} \mathrm{erg}$. Equating this to $G M_{\star} M_{\mathrm{acc}} / R_{\star}$ one obtains (assuming $M_{\star}=1.0 M_{\odot}$ and $R_{\star}=1.0 R_{\odot}$ ) $M_{\text {acc }} \simeq 10^{-5} M_{\odot}$. Note that this estimate does not take into account the energy radiated away before the discovery (it is very likely that the eruption of V4332 Sgr started well before 28 February 1994), nor the energy stored in the matter now circulating the central star, nor the kinetic energy in mass loss. Given the above estimate as well as that of $M_{\mathrm{e}}$ in Sect. 5, we can conclude that the mass of the accreted object was $\gtrsim 10^{-4} M_{\odot}$.

The unusual emission line spectrum observed in April 2003 and described in Sect. 3 deserves futher studies as it can provide important insight into the current state of V4332 Sgr. From our preliminary analysis in Sect. 3 we can conclude that it must originate in an optically thin, neutral, molecular, cold medium. Low optical thickness means low column density which, with large equivalent widths of many lines in the spectrum, requires large volume - considerably larger than that of the central star. This obviously rules out the stellar atmosphere and regions in its near vicinity. The low rotational temperature inferred in Sect. 3 (from different emission bands) implies that the medium, in the bulk, is significantly cooler than $1000 \mathrm{~K}$. This is the sort of temperature we have found for the disc-like structure in Sect. 6. However, this structure is expected to be mostly optically thick. It seems that possible sites for the emision line spectrum to originate might be in regions between the stellar surface and the disc where the contracting stellar envelope might have left some matter circulating now near the equatorial plane. BA04 have found that the observed width of the KI emission lines if interpreted with Doppler broadening give a velocity of $260 \mathrm{~km} \mathrm{~s}^{-1}$. The measured $F W H M$ of the $\mathrm{CaI}$ line in our spectrum (see Table 2) when corrected for the 
instrumental $F W H M(5.6 \AA)$ gives a similar value, i.e. $280 \mathrm{~km} \mathrm{~s}^{-1}$. This value would correspond to a Keplerian velocity at $\sim 10 R_{\odot}$ for a $1 M_{\odot}$ star. The required excitation could result from the stellar radiation or viscous processes, or both.

The galactic position of V4332 Sgr, $l=13^{\circ} .63 b=-9^{\circ} .40$, might suggest that the object is related to the Galactic bulge. Thus V4332 Sgr would be at a distance of $\sim 8.5 \mathrm{kpc}$, which, on the one hand, seems to be too large for the observed redenning, as discussed in Sect. 4.2. On the other hand, however, it would be easier in this case to understand the observed radial velocity of V4332 Sgr, which is, as discussed in Sect. 6, inconsistent with the rotation of the Galactic disc. At a distance of $8.5 \mathrm{kpc}$ all the radii and luminosities derived in this paper would increase by a factor of 4.7 and 22 , respectively. In particular, the progenitor would be a $\mathrm{G}$ type subgiant of a mass of $\sim 2 M_{\odot}$, as can be inferred from stellar evolutionary tracks (e.g. Iben 1965), evolving from the main sequence to the red giant branch. The star would have a $\sim 0.2 M_{\odot}$ helium core and an envelope with a mean density of $0.02 \mathrm{~g} \mathrm{~cm}^{-3}$. Thus one could refer to the scenario of Retter \& Marom (2003) while interpreting the eruption of V4332 Sgr. Indeed $M_{\text {acc }} \simeq 10^{-4} M_{\odot}$ is then required to explain the energy emitted in the eruption so a massive planet or a brown dwarf would be involved in the merger event. However, the accreted object, being significantly denser than the subgiant envelope, would be expected to penetrate deeply in the envelope, as discussed e.g. in Livio \& Soker (1984). Thus most of the subgiant envelope would be disturbed and the whole event, the contraction phase in particular, would procede on a much longer time scale than observed. Indeed, modelling analogous to that in Sect. 5 but done to match the observed increase of the radius by a factor of 4.7 , assuming a central star mass of $2 M_{\odot}$, requires $M_{\mathrm{e}} \simeq 3 \times 10^{-4} M_{\odot}$. In the discussed case we would rather expect $M_{\mathrm{e}} \simeq 1 M_{\odot}$ to be involved. Note that Livio \& Soker (1984) show that a merger event in the case of a giant and a massive planet may last several thousands years.

There is no doubt that futher detailed observations of V4332 Sgr are necessary. Photometric and spectroscopic measurements in the near and far infrared would be of particular value as the object is emitting most of its energy in this wavelength range. High quality spectroscopy in the optical would also be very important for understanding the origin of the unusual emission-line spectrum as well as for analysing the underlying stellar component. In particular, high resolution spectroscopy in the atomic lines would allow precise determination of the radial velocity of the object, as well as investigation of the kinematic structure of the emitting region. The object is probably evolving on a time scale of years so long term observational monitoring should be undertaken. We hope that the analysis of the existing observational data in this paper, followed by our interpretation and conclusions (which in several points may be regarded as speculative) will stimulate deeper astrophysical interest in V4332 Sgr, V838 Mon and, possibly, other objects of similar nature.

Acknowledgements. The research reported in this paper has partly been supported from a grant No. 2.P03D.002.25 financed by the Polish State Committee for Scientific Research.

\section{References}

Acker, A., Ochsenbein, F., Stenholm, B., et al. 1992, Strasbourg-ESO Catalogue of Galactic Planetary Nebulae (ESO Publication)

Avedisova, V. S. 2002, AZh, 79, 216

Banerjee, D. P. K., Varricatt, W. P., Ashok, N. M., \& Launila, O. 2003, ApJ, 598, L31 (BVA03)

Banerjee, D. P., \& Ashok, N. M. 2004, ApJ, 604, L57 (BA04)

Banerjee, D.P., Varricatt, W. P., \& Ashok, N. M. 2004, ApJ, 615, L53 (BVA04)

Barnbaum, C., Omont, A., \& Morris, M. 1996, A\&A, 310, 259

Bessell, M. S., \& Brett, J. M. 1988, PASP, 100, 1134

Brand, J., \& Blitz, L. 1993, A\&A, 275, 67

Crause, L. A., Lawson, W. A., Kilkenny, D., et al. 2003, MNRAS, 341,785

Drilling, J. S., \& Landoldt, A. U. 2000, in Allen's Astrophysical Quantities, the 4th edition, ed. A. N. Cox (New York: Springer-Verlag), 381

Dame, T. M., Hartmann, D., \& Thaddeus, P. 2001, ApJ, 547, 792

Frank, J., King, A., \& Reine, D. 2002, Accretion Power in Astrophysics, Third edition (Cambridge Univeristy Press)

Gilmore, A. C. 1994, IAU Circ., 5943, 5944, 5949

Herbig, G. H. 1974, ApJ, 188, 533

Iben, I. 1965, ApJ, 141, 993

Johnson, H. L. 1966, ARA\&A, 4, 193

Koornneef, J. 1983, A\&A, 128, 84

Kurucz, R. 1999, CDROM No. 24

Lee, T. A. 1970, ApJ, 162, 217

Livio, M., \& Soker, N. 1984, MNRAS, 208, 763

Lynds, B. T. 1965, ApJS, 12, 163

Martini, P., Wagner, R. M., Tomaney, A., et al. 1999, AJ, 118, 1034 (MWT99)

Mould, J., Cohen, J., Graham, J. R., et al. 1990, ApJ, 353, L35

Munari, U., Henden, A., Kiyota, S., et al. 2002, A\&A, 389, L51

Retter, A., \& Marom, A. 2003, MNRAS, 345, L25

Russeil, D. 2003, A\&A, 397, 133

Schmidt-Kaler, Th. 1982, Landolt-Börnstein: Numerical Data and Functional Relationships in Science and Technology, ed. K. Schaifers, \& H. H. Voigt (Berlin: Springer-Verlag), VI/2b

Schwenke, D. W. 1998, Chemistry and Physics of Molecules and Grains in Space, Faraday Discussions No. 109, 321

Sharpless, S. 1959, ApJS, 4, 257

Skiff, B. A. 1994, IAU Circ., 5944

Soker, N., \& Tylenda, R. 2003, ApJ, 582, L105

Tylenda, R. 2005, A\&A, 436, 1009

Tylenda, R., Acker, A., Stenholm, B., \& Köppen, J. 1992, A\&AS, 95, 337 